\title{
Preparatory Experiments on Oscillatory Thermocapillary bubble Convection
}

\author{
Ebram Tadrous ${ }^{1,2}$, Günter Wozniak ${ }^{1,3}$ \\ ${ }^{1}$ Chemnitz University of Technology, Faculty of Mechanical Engineering, Institute of Mechanics and Thermodynamics, \\ Chair of Fluid Mechanics \\ Reichenhainer Str. 70, D-09107 Chemnitz, Germany \\ Ebram.boshra.hanna_tadrous@mb.tu-chemnitz.de; Guenter.wozniak@mb.tu-chemnitz.de \\ ${ }^{2}$ Cairo University, Faculty of Engineering, Chair of Mechanical Power Engineering \\ Giza, Egypt \\ ${ }^{3} \mathrm{Suvis} \mathrm{GmbH}$ \\ Altchemnitzer Str. 11, D-09120 Chemnitz, Germany
}

\begin{abstract}
Thermocapillary-driven convection around a bubble under a heated wall is experimentally investigated under gravitational conditions. The induced flows are categorized into laminar, periodic oscillatory, and non-periodic oscillatory flow through the pattern of the tracer particle motion around the bubble using particle image velocimetry PIV, and through surface temperature variation using resistance temperature detectors (RTDs). Transient temperature development at a discrete point can reflect the fluctuations of the flow. Chaotic flows are observed in these experiments. They are distinguished from the periodic oscillatory flow through the non-periodicity of temperature fluctuations along a certain point around the bubble within the flow domain.
\end{abstract}

Keywords: Marangoni convection- PIV- Temperature and flow fluctuations- Tracer particles- Bubble

\section{Introduction}

Temperature gradients in liquid-liquid and liquid-gas systems evolve both free and forced convection influencing heat and mass transfer processes within the system. On earth, the convective flow due to buoyancy is the dominant flow, which is a result of density variations due to temperature gradients in the flow domain. Moreover, a non-neglected secondary convective flow is observed on the phase boundary between two liquids or liquid and gas. This additional convective flow is a result of temperature gradients at the phase boundary. The interfacial surface tension gradients $\partial \sigma / \partial \mathrm{T}$ caused by interfacial temperature gradients $\partial \mathrm{T} / \partial \mathrm{Z}$ lead to a fluid flow towards regions of high surface tensions and low temperatures well-known as thermocapillary Marangoni convection. The physical problem is mainly characterized by the Marangoni number, $\mathrm{Mg}$, representing the ratio of convective heat flow induced by capillary convection to the heat transfer due to conduction. Many authors defined the Mg-number in different forms, depending on both thermal diffusivity $\alpha$ and dynamic viscosity $\eta$ of liquid, as shown in table 1 . Wozniak et al. [1,2] used the bubble radius $R_{B I}$ only to represent the characteristic length in calculating the dimensionless number $\mathrm{Mg}$, while Chun et al. [3] used both bubble diameter and its vertical expansion B. Chun et al. [3] used an empirical equation to calculate the vertical temperature gradient near the bubble in terms of the upper plate temperature $T_{D}$ and lower plate temperature $T_{B}$. Wozniak et al. [2] used a defined temperature gradient between the upper plate and a reference point $\mathrm{T}_{\text {ref, }}$ where $\mathrm{r}_{\text {ref }}$ is the radial distance away from the centre of the upper plate, and $\Delta \mathrm{z}$ ref is the vertical distance downwards the upper plate, while in [1] the mean whole temperature gradient along the liquid matrix $|\partial \mathrm{T} / \partial \mathrm{Z}| \propto$ is simply considered. In this work, the form used by Chun et al. [3] is used, as both geometrical parameters of the bubble are considered. Moreover, the actual temperature gradient near the bubble is used in the equation instead of the whole temperature gradient of the test fluid. The shape of the injected bubble is affected by buoyancy force. It is noticed that there is no geometrical bubble shape similarity among different bubble radii in our experiments, as shown in table 2. Marangoni convection can dominate heat and mass transfer more under microgravity conditions. Therefore, manufacturers should consider that thermocapillary convection in material production processes in space [4]. Moreover, one can purposefully use the temperature gradient to eliminate or move bubbles or drops suspended in liquid metals [5], as well as in other applications 
like manufacturing of single-walled carbon nanotubes [6], monocrystal production, and investigating Flame spread above liquid fuels [7], to mention only a few examples.

Table 1: Definition of the dimensionless number Mg by different Authors.

\begin{tabular}{|c|c|}
\hline Wozniak et al. [1] & $M g=\frac{R_{B L}^{2}}{\alpha \eta}\left|\frac{\partial \sigma}{\partial T}\right|\left|\frac{\partial T}{\partial Z}\right| \infty$ \\
\hline Chun et al. [3] & $\begin{array}{c}\boldsymbol{M g}=\frac{\boldsymbol{R}_{\boldsymbol{B L}} \boldsymbol{B}}{\alpha \boldsymbol{\eta}}\left|\frac{\partial \sigma}{\partial \boldsymbol{T}}\right|\left|\frac{\partial T}{\partial Z}\right| r e f \\
\left|\frac{\partial T}{\partial Z}\right| \mathrm{ref}=0.03717\left(\mathrm{~T}_{\mathrm{D}}-\mathrm{T}_{\mathrm{B}}\right), \Delta \mathrm{z}_{\text {ref }}=1 \mathrm{~mm}, \mathrm{r}_{\text {ref }}=45 \mathrm{~mm}\end{array}$ \\
\hline Wozniak et al. [2] & $\begin{array}{c}M g=\frac{\boldsymbol{R}_{\boldsymbol{B L}}^{2}}{\boldsymbol{\alpha} \boldsymbol{\eta}}\left|\frac{\partial \boldsymbol{\sigma}}{\boldsymbol{\partial T}}\right|\left|\frac{\boldsymbol{T D}-\boldsymbol{T r e f}}{\Delta \boldsymbol{Z} r e f}\right| \\
\Delta \mathrm{z}_{\mathrm{ref}}=7 \mathrm{~mm}, \mathrm{r}_{\mathrm{ref}}=45 \mathrm{~mm}\end{array}$ \\
\hline
\end{tabular}

Table 2: The relation between the radius of the injected air bubble $\mathrm{R}_{\mathrm{Bl}}(\mathrm{mm})$ and its vertical expansion $\mathrm{B}(\mathrm{mm})$ for silicone oil $A K$ 0.65.

\begin{tabular}{|c|c|c|}
\hline $\mathrm{R}_{\mathrm{BL}}(\mathrm{mm})$ & $\mathrm{B}(\mathrm{mm})$ & $\mathrm{R}_{\mathrm{BL}} / \mathrm{B}$ \\
\hline 2.03 & 2.25 & 0.9 \\
\hline 4.1 & 2.35 & 1.74 \\
\hline 4.71 & 2.54 & 1.85 \\
\hline 6 & 2.69 & 2.23 \\
\hline 6.2 & 2.69 & 2.3 \\
\hline 6.33 & 2.69 & 2.35 \\
\hline 8.51 & 2.77 & 3.07 \\
\hline
\end{tabular}

Many efforts have been made to study Marangoni convection, also to determine the behaviour of the flow, and the factors leading to instabilities of that flow like periodic oscillations or non-periodic ones till it turns to turbulent flow, [8], [9], [3], and [10]. Therefore, our work aims at developing a test cell for high Mg-numbers to study the flow behaviour of the examined volatile fluid, where high non-periodic oscillations are achieved. From the previously examined liquids Methanol, Ethanol, and Silicon oil AK 0.65 are the most suitable liquids to reach high Mg-numbers.

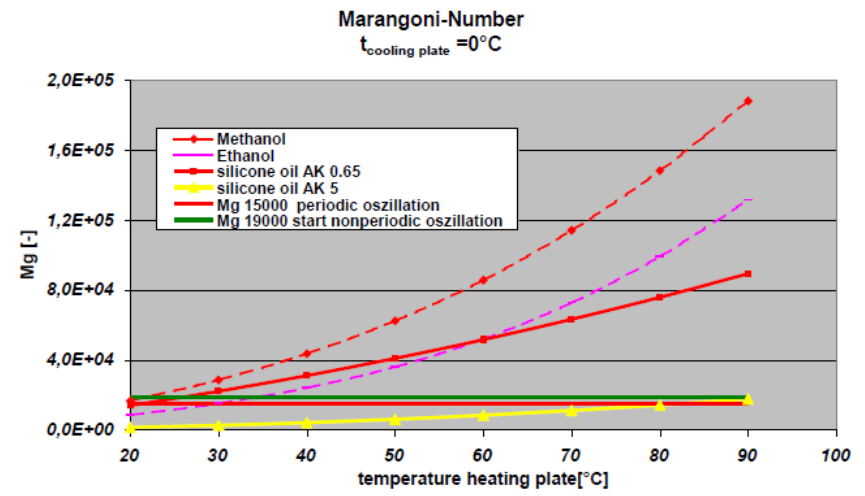

Fig. 1: Mg-numbers using silicone oil and alcohol, bubble radius of $4 \mathrm{~mm}$ in all cases [11]. 


\section{Experiments Setup and Methodology}

In order to systematically investigate and prove the turbulent thermocapillary flow around a bubble under a heated wall wall by optical measurement techniques, a certain adjustment of the test cell is being used to pursue that experimental study, study, as shown in figure 2. The test used is quite similar to that described by Schade et al. [11]. It is, in brief, a rectangular rectangular cavity ending with two horizontal copper plates each of $50 \mathrm{X} 80 \mathrm{~mm}$ as horizontal dimensions. These copper plates are maintained at two different uniform temperatures to generate the vertical temperature gradient required for generating the thermocapillary flow. The lower plate is maintained at a lower temperature, $\mathrm{T}_{\mathrm{u}}$, by means of a Compact LowTemperature Thermostat, while the other upper plate is maintained at a higher temperature, $\mathrm{T}_{\mathrm{o}}$, using an electrical resistance heater. To enhance the visibility, the front and back side walls are made of optical glass. Three more copper plates are mounted on the lower copper base to decrease the clear height of the liquid matrix to $15 \mathrm{~mm}$ in order to achieve higher temperature gradients among the test cell.

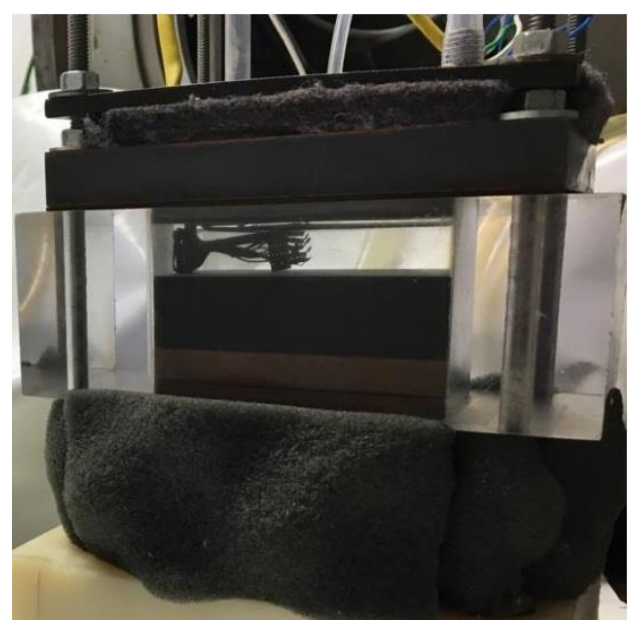

Fig. 2: Structure of test cell.

Low viscous Silicon oil Ak 0.65 is used as a test liquid with a descriptive adjustment of an argon-iron laser to record streak images of the flow using Borosilicate particles of $10 \mu \mathrm{m}$ in diameter as tracer particles. With that, various patterns of flow field can be observed. For temperature measurement and control, six thermo-elements (RTDs) are inserted vertically at different distances from the upper plate near the midline to measure the actual temperature gradient near the bubble. After heating the upper plate and cooling the lower one as long until the stable temperature gradient along the liquid is reached, the bubble is injected from the central hole in the upper plate. Later the flow is visualized and the data are processed to find out both velocity and temperature behaviours. The liquid temperature at a discrete point $A(R=8.5 \mathrm{~mm}, \mathrm{~S}=1 \mathrm{~mm})$ near the upper plate is measured every $100 \mathrm{~ms}$, where $\mathrm{R}$ is the radial distance away from the centre of the upper plate, and $\mathrm{S}$ is the vertical distance downwards the upper plate. So that one can check the periodicity of temperature fluctuations, which was recommended to achieve a better accuracy in recording the wave period [9].

Measuring the temperature fluctuations at the discrete point $\mathrm{A}$ is considered as an indication of the periodicity of the oscillating liquid particles. The frequency of the temperature fluctuations of $0.4 \mathrm{~Hz}$, as shown in figure 3 agrees with the calculated oscillating frequency of the fluid particles moving around the bubble. The mentioned oscillatory frequency of the fluid particles is determined by tracing the movement of a single particle with the help of 27 consecutive PIV camera shots of the flow field around the injected air bubble, with a total time of $3.375 \mathrm{secs}$, as shown in figure 4 . 


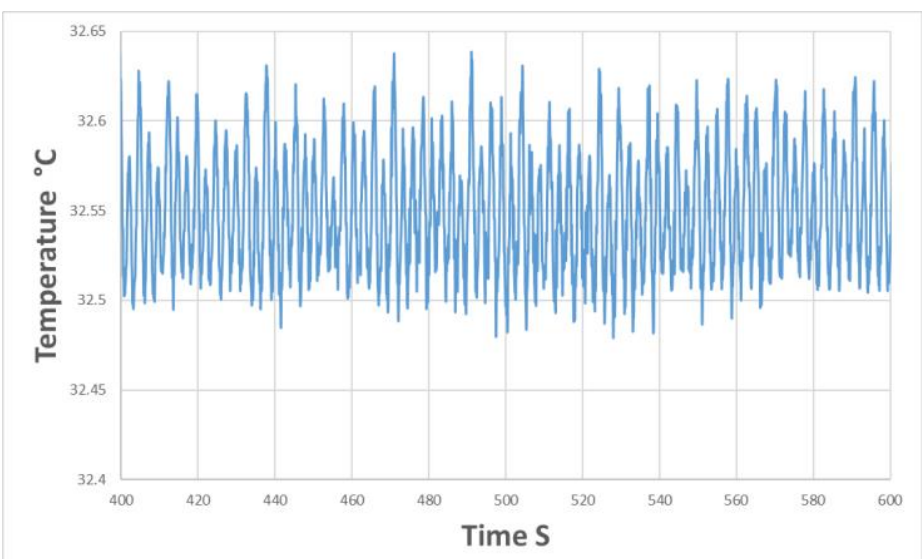

Fig. 3: Transient development of temperature at the discrete point $\mathrm{A}$ in the liquid, $\mathrm{Mg}=41240, \mathrm{R}_{\mathrm{Bl}}=6 \mathrm{~mm}, \mathrm{~B}=2.69 \mathrm{~mm}, \mathrm{~T}_{0}=36.9^{\circ} \mathrm{C}$, $\mathrm{T}_{\mathrm{u}}=2{ }^{\circ} \mathrm{C}$.

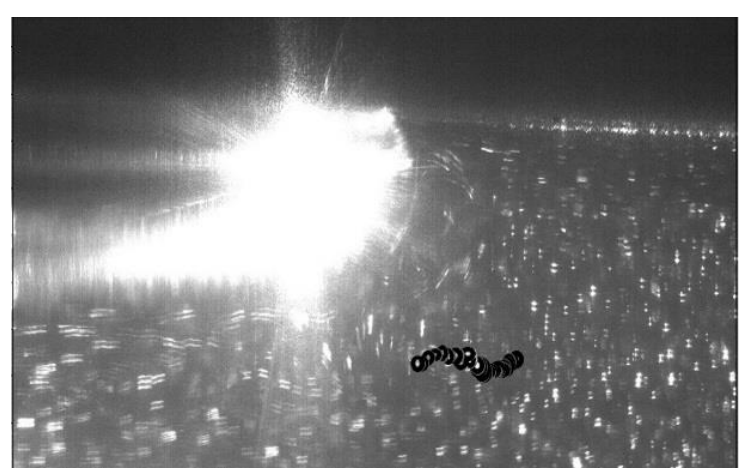

Fig. 4: Tracing of a single particle in the flow field around the bubble, $\mathrm{Mg}=41240, \mathrm{R}_{\mathrm{Bl}}=6 \mathrm{~mm}, \mathrm{~B}=2.69 \mathrm{~mm}, \mathrm{~T}_{\mathrm{o}}=36.9^{\circ} \mathrm{C}, \mathrm{T}_{\mathrm{u}}=2{ }^{\circ} \mathrm{C}$.

\section{Results and Discussions}

At low Mg-numbers, liquid particles around the bubble periphery are attracted downwards to regions of low temperature and high surface tension, and then pushed upwards by buoyancy forces forming a thin anticlockwise vortex near the bubble surface, as shown in figure 5. Figure 6 shows the temporal development of temperature at a discrete point A. Low amplitude temperature variations of $0.03{ }^{\circ} \mathrm{C}$ at the same point ensure a steady vertical temperature gradient along the test cell. These low amplitude variations are caused by temperature sensor self-instabilities. An instantaneous increase in temperature is observed, once the bubble is injected. Temperature oscillations with a low frequency of 0.1 $\mathrm{Hz}$ of temperature take about 7 minutes and then it reaches a plateau of $26.25{ }^{\circ} \mathrm{C}$ with very low oscillations which can be neglected.

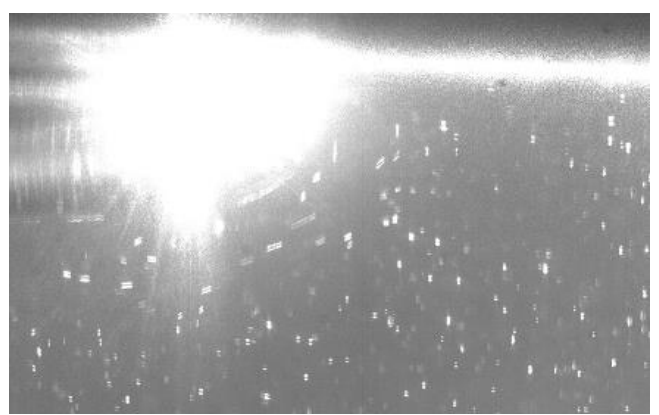

Fig. 5: Flow field around the bubble, $\mathrm{Mg}=12271, \mathrm{R}_{\mathrm{Bl}}=8 \mathrm{~mm}, \mathrm{~B}=2.75 \mathrm{~mm}, \mathrm{~T}_{\mathrm{o}}=26.9^{\circ} \mathrm{C}, \mathrm{T}_{\mathrm{u}}=16.4{ }^{\circ} \mathrm{C}$. 


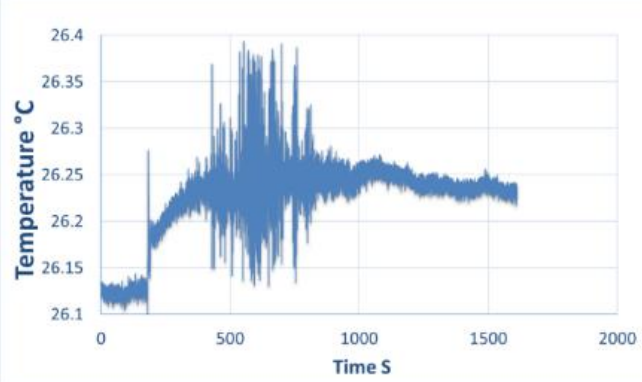

Fig. 6: Transient development of temperature at the discrete point $\mathrm{A}$ in the liquid, $\mathrm{Mg}=12271, \mathrm{R}_{\mathrm{Bl}}=8 \mathrm{~mm}, \mathrm{~B}=2.75 \mathrm{~mm}, \mathrm{~T}_{0}=26.9^{\circ} \mathrm{C}$, $\mathrm{T}_{\mathrm{u}}=16.4^{\circ} \mathrm{C}$.

In low Mg-number experiments, the effect of changing bubble diameter is also discussed. It is found that the duration of temperature fluctuations accompanied by bubble injection increases with injecting larger bubbles, however, the span of temperature oscillations remains the same as long as the temperature gradient along the test cell is constant, as shown in figure 7. One can notice an increase of the mean temperature in case of the larger bubble, due to the injection of more amount of air to the bubble.
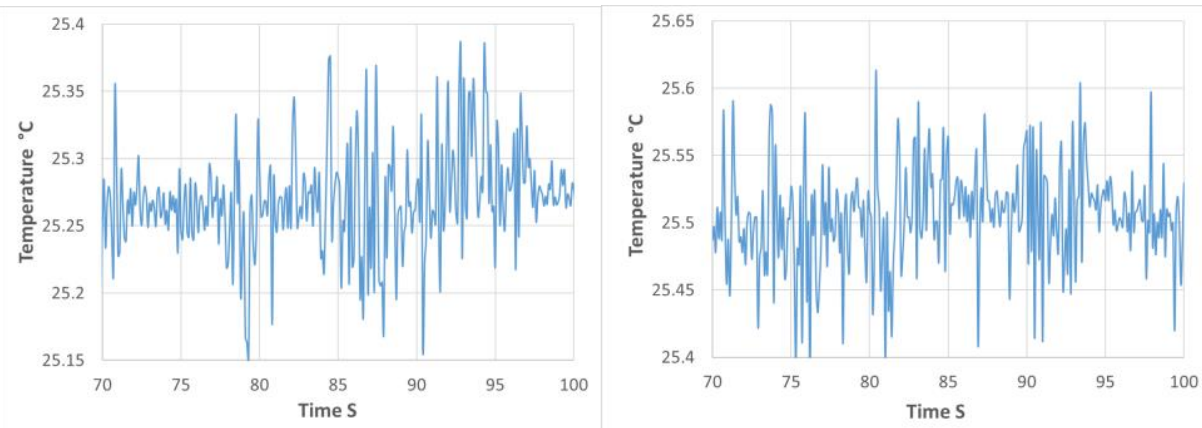

Fig. 7: Comparison between transient development of temperature at the discrete point $\mathrm{A}$ in the liquid, $\mathrm{T}_{\mathrm{o}}=26.9{ }^{\circ} \mathrm{C}, \mathrm{T}_{\mathrm{u}}=10.1^{\circ} \mathrm{C}$, (left, $\mathrm{Mg}=19139, \mathrm{R}_{\mathrm{Bl}}=7 \mathrm{~mm}, \mathrm{~B}=2.71 \mathrm{~mm}$ ), (right, $\mathrm{Mg}=22196, \mathrm{R}_{\mathrm{Bl}}=8 \mathrm{~mm}, \mathrm{~B}=2.75 \mathrm{~mm}$ ).

Further analysis of the temporal temperature variations at a discrete point around the bubble can help to evaluate the behaviour of the flow [3]. Figure 8 shows laminar flow fields around the injected bubble at different low $\mathrm{Mg}$-number conditions, where no obvious temperature oscillations of amplitude higher than $0.03{ }^{\circ} \mathrm{C}$ appear. Therefore, the flow is considered as laminar being. Regarding the flow field behaviour, a secondary vortex does not appear at such a lower $\mathrm{Mg}-$ number, as shown in figure 8, while it starts to appear at relatively higher Mg-numbers.
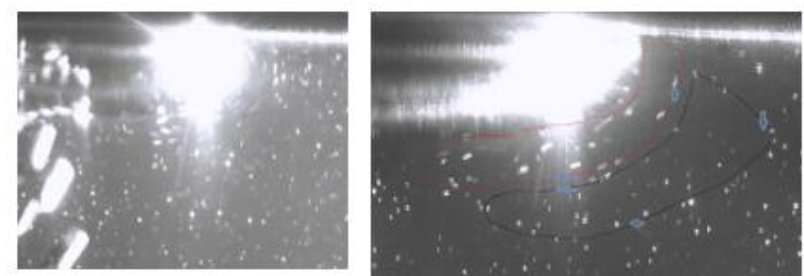

Fig. 8: Flow field around the bubble, (left, $\mathrm{Mg}=6746, \mathrm{R}_{\mathrm{Bl}}=5.5 \mathrm{~mm}, \mathrm{~B}=2.63 \mathrm{~mm}, \mathrm{~T}_{\mathrm{o}}=27.3{ }^{\circ} \mathrm{C}, \mathrm{T}_{\mathrm{u}}=18^{\circ} \mathrm{C}$ ), (right, $\mathrm{Mg}=10260, \mathrm{R}_{\mathrm{BI}}=8$ $\left.\mathrm{mm}, \mathrm{B}=2.75 \mathrm{~mm}, \mathrm{~T}_{\mathrm{o}}=27.3^{\circ} \mathrm{C}, \mathrm{T}_{\mathrm{u}}=18^{\circ} \mathrm{C}\right)$.

While further increasing the temperature gradient along the test cell at higher Mg-numbers, the flow field around the bubble remains the same till reaching $\mathrm{Mg}=18355$, where periodic oscillations of the temperature of a low frequency of 0.2 
$\mathrm{Hz}$ at the discrete point around the bubble as shown in figure 9 appear. Furthermore, the small variations of amplitude of $0.03{ }^{\circ} \mathrm{C}$ are also neglected and considered as noise in the temperature measurement. At this $\mathrm{Mg}$ - number, the first vortex is enlarged near the upper plate as shown in figure 10.

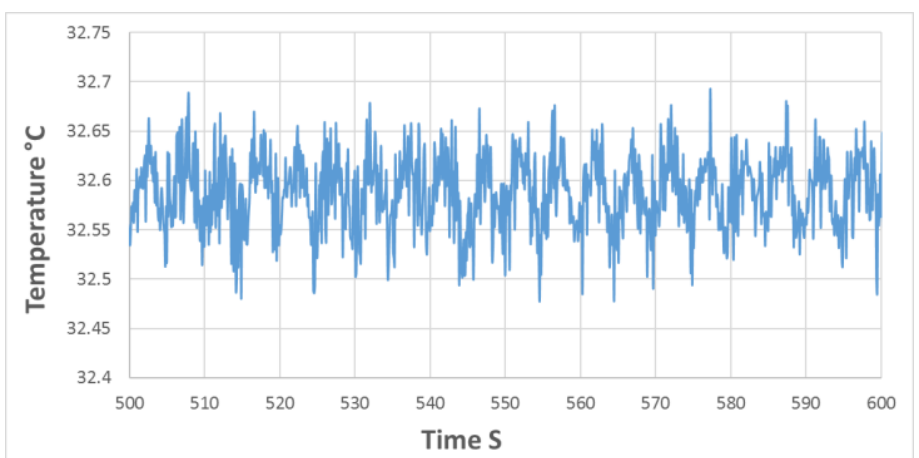

Fig. 9: Transient development of temperature at the discrete point $\mathrm{A}$ in the liquid, $\mathrm{Mg}=18355, \mathrm{R}_{\mathrm{Bl}}=6.05 \mathrm{~mm}, \mathrm{~B}=2.69 \mathrm{~mm}, \mathrm{~T}_{\mathrm{o}}=35.6$ ${ }^{\circ} \mathrm{C}, \mathrm{T}_{\mathrm{u}}=18{ }^{\circ} \mathrm{C}, \mathrm{dT} / \mathrm{dz}=0.79{ }^{\circ} \mathrm{C} / \mathrm{mm}$.

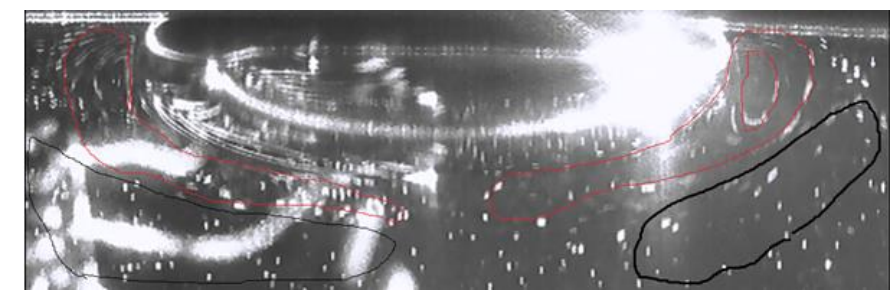

Fig. 10: Flow field around the bubble, $\mathrm{Mg}=18355, \mathrm{R}_{\mathrm{Bl}}=6.05 \mathrm{~mm}, \mathrm{~B}=2.69 \mathrm{~mm}, \mathrm{~T}_{\mathrm{o}}=35.6{ }^{\circ} \mathrm{C}, \mathrm{T}_{\mathrm{u}}=18{ }^{\circ} \mathrm{C}, \mathrm{dT} / \mathrm{dz}=0.79{ }^{\circ} \mathrm{C} / \mathrm{mm}$.

The mentioned case of periodically oscillating flow of $\mathrm{Mg}=18355$ matches that result mentioned before by Raake et al. [10], as oscillations were supposed to arise starting from a temperature gradient of $0.5^{\circ} \mathrm{C} / \mathrm{mm}$, followed by high degree of oscillations at around of $1{ }^{\circ} \mathrm{C} / \mathrm{mm}$. According to figure 11 , the discussed case of $\mathrm{Mg}=18355$, and $\mathrm{r}_{\mathrm{B}} / \mathrm{z}_{\mathrm{B}}$, ratio of bubble radius to its vertical expansion $=2.25$, was expected by Chun et al. al [3] to oscillate periodically. This matches our results.

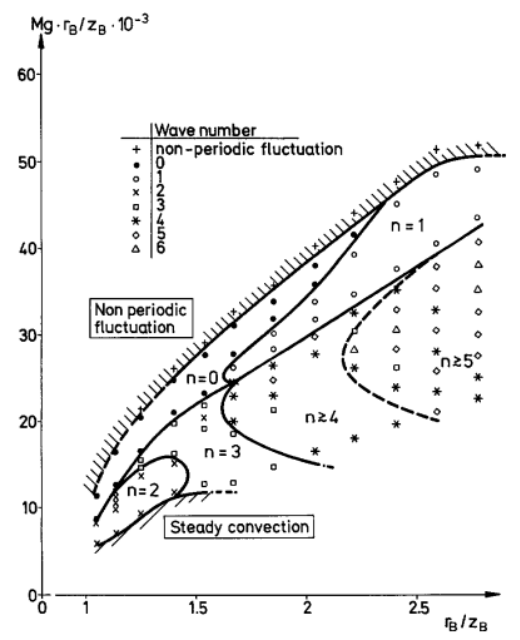

Fig. 11: Mode selection on the plane of the modified Mg-number vs. the bubble geometry parameter $\mathrm{r}_{\mathrm{B}} / \mathrm{z}_{\mathrm{B}}$ [3]. 
Further experiments were performed at higher Marangoni values. With higher temperature gradients, higher orders of temperature oscillations are noticed. Also a certain development in the flow field around the bubble has been observed. The primary vortex splits into more eddies forming three vortices around the bubble, and the fluid tracer particles are found to be oscillating, which matches also with the experimental work of Raake et al. [10], figure 12.

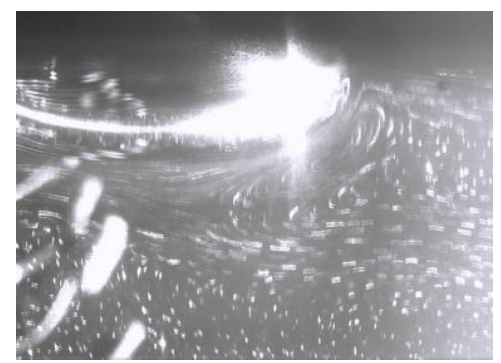

Fig. 12: Flow field around the bubble, $\mathrm{Mg}=38550, \mathrm{R}_{\mathrm{Bl}}=5.5 \mathrm{~mm}, \mathrm{~B}=2.63 \mathrm{~mm}, \mathrm{~T}_{\mathrm{o}}=47.9^{\circ} \mathrm{C}, \mathrm{T}_{\mathrm{u}}=-3.11^{\circ} \mathrm{C}, \mathrm{dT} / \mathrm{dz}=1.87^{\circ} \mathrm{C} / \mathrm{mm}$.

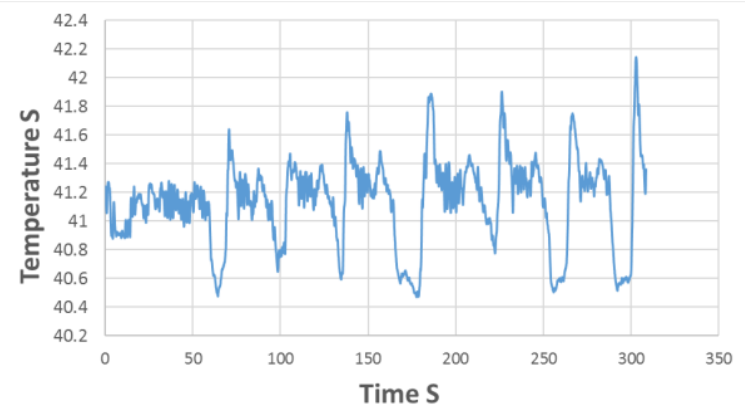

Fig. 13: Transient development of temperature at the discrete point $A$ in the liquid, $M g=38550, R_{B l}=5.5 \mathrm{~mm}, B=2.63 \mathrm{~mm}, T_{0}=47.9$ ${ }^{\circ} \mathrm{C}, \mathrm{T}_{\mathrm{u}}=-3.11^{\circ} \mathrm{C}, \mathrm{dT} / \mathrm{dz}=1.87^{\circ} \mathrm{C} / \mathrm{mm}$.

As predicted, the temperature at the discrete point oscillates in a non-periodic form with a higher frequency of $0.64 \mathrm{~Hz}$ and a higher amplitude of $0.25{ }^{\circ} \mathrm{C}$. However, sudden consequent decrease and increase in temperature are noticed. This sudden change repeats itself periodically every 35 seconds, as shown in figure 13 . That is attributed to the periodic movement of the second vortex in the vertical direction, as shown in figure 14, or possibly due to unwanted vibrations of the injected bubble during the experiment.

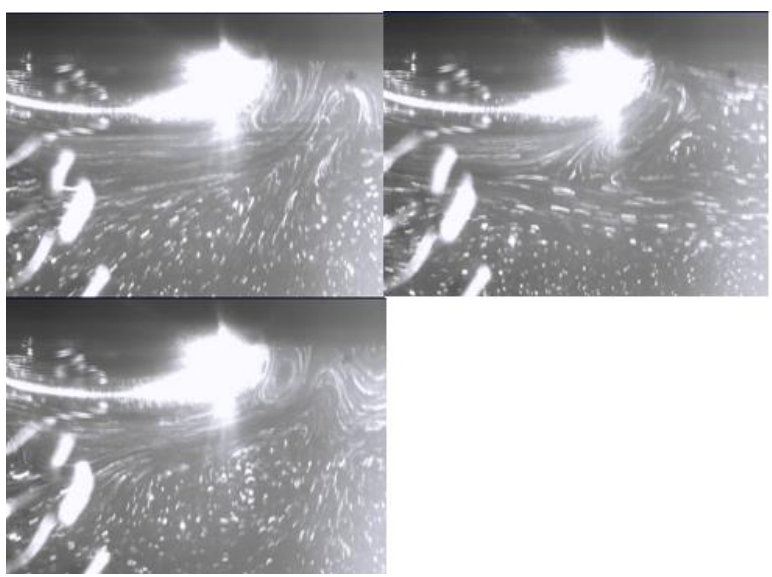

Fig. 14: Flow field around the bubble at simultaneous captures, where: $\mathrm{Mg}=38550, \mathrm{R}_{\mathrm{Bl}}=5.5 \mathrm{~mm}, \mathrm{~B}=2.63 \mathrm{~mm}, \mathrm{~T}_{\mathrm{o}}=47.9^{\circ} \mathrm{C}, \mathrm{T}_{\mathrm{u}}=-3.11$ ${ }^{\circ} \mathrm{C}, \mathrm{dT} / \mathrm{dz}=1.87^{\circ} \mathrm{C} / \mathrm{mm}$. 


\section{Conclusion}

Transient temperature development at a discrete point can reflect the fluctuations of the Marangoni flow. At numbers of around 18355, periodic oscillations of AK 0.65 particles around the injected bubbles are observed. With increasing the temperature gradient along the injected bubble surface, the primary vortex splits into more vortices. Accordingly, at higher Ma- numbers, the degree of oscillations increases, represented by higher amplitudes and of temperature oscillations. Among all the pursued experimental work, the oscillation frequency was not reproducible in all experiments. That agrees with both Chun et al. [3] and Betz et al. [9]. The mode of oscillation does not depend only on the known parameters affecting the formed Marangoni flow around the bubble, but also another disturbance like the vibration of the bubble and the initial thermal and mechanical conditions while forming the bubble as mentioned before [3]. Increasing the Temperature of the heated plate is limited to the vapour pressure of AK 0.65. According to Vapour pressure- Temperature curves of AK 0.65, it is theoretically noticed that a gauge pressure of 1 bar is enough to shift the vaporization temperature to the range near $100{ }^{\circ} \mathrm{C}$. Therefore, it is recommended to pursue the experiments under gauge pressure.

\section{References}

[1] G. Wozniak and K. Wozniak, "Buoyancy and thermocapillary flow analysis by the combined use of liquid crystals and PIV," Experiments in Fluids, vol. 17, pp. 141-146, 1994.

[2] K. Wozniak, G. Wozniak, and T. Rösgen, "Particle-image-velocimetry applied to thermocapillary convection," Experiments in Fluids, vol. 10, pp. 12-16, 1990.

[3] C. H. Chun, D. Raake, and G. Hansmann, "Oscillating convection modes in the surroundings of an air bubble under a horizontal heated wall," Exp. Fluids., vol. 11, pp. 359-367, 1991.

[4] J. Aleksic, J. A. Szymczyk, A. Leder, and T. A. Kowalewski, "Experimental investigations on thermal, thermocapillary and forced convection in Czochralski crystal growth configuration," WIT Transactions on Modelling and Simulation, vol. 30 , no. $10,2001$.

[5] G. Amberg and J. Shiomi, "Thermocapillary Flow and Phase Change in Some Widespread Materials Processes," FDMP, vol. 1, pp. 81-95, 2005.

[6] S. H. Jin, S. N. Dunham, and J. Song, "Using nanoscale thermocapillary flows to create arrays of purely semiconducting single-walled carbon nanotubes," Nature Nanotechnology, vol. 8, pp. 347-355, 2013.

[7] Cai. J. Jinsheng Cai, F. Liu, and W. A. Sirignano, "Opposed Flow Impact on Flame Spread Above Liquid Fuel Pools," in Proceedings of the US Western States Section of the Combustion Institute, Spring Meeting, 2002.

[8] H. G. Heiland, K. P. Schade, and G. Wozniak, "Thermocapillary flow on a gas-liquid surface under normal and reduced gravity," PAMM, Proc. Appl. Math. Mech., vol. 11, pp. 609-610, 2011.

[9] J. Betz and J. Straub, "Numerical and experimental study of the hat transfer and fluid flow by thermocapillary convection around gas bubbles," Heat and Mass Transfer, vol. 37, pp. 215-227, 2001.

[10] D. Raake, J. Siekmann, and C. H. Chun, "Temperature and velocity fields due to surface tension driven flow," Experiments in Fluids, vol. 7, pp. 164-172, 1989.

[11] K. P. Schade, G. Wozniak, and Ch. Gessenhard, "Experimental investigation of thermocapillary flow at the phase boundary of a bubble in a liquid," in Proceedings of the 6th International Conference on Multiphase FI, 2007. 